Letters to the Editor

\title{
Ischemic Stroke in the COVID-19 Patient
}

\author{
${ }^{1}$ Ivan Lozada-Martinez, ${ }^{1}$ Daniela M. Torres-Llinás, ${ }^{1}$ Luis R. Moscote-Salazar, \\ ${ }^{2}$ Sabrina Rahman, ${ }^{3} \mathrm{AHM}$ Ataullah, ${ }^{4}$ Nazmul Huda Ridoy and ${ }^{5}$ Moshiur Rahman
}

\author{
${ }^{1}$ School of Medicine, University of Cartagena, Colombia \\ ${ }^{2}$ Department of Public Health, Independent University-Bangladesh, Bangladesh \\ ${ }^{3}$ Medical Officer, Sher-E-Bangla Medical College Hospital, Bangladesh \\ ${ }^{4}$ School of Medicine, Jahurul Islam Medical College, Bangladesh \\ ${ }^{5}$ Department of Neurosurgery, Holy Family Red Crescent Medical College, Bangladesh
}

Currently, there are many questions around the real impact of COVID-19 at an organic level and the resolution of these is one of the main objectives of the scientific societies and public health institutions around the world, to establish the most effective and safe management protocol in the approach of this disease (Qureshi et al., 2020). Systematic reviews and metaanalyses mention that the common presentation of this entity is based on a syndromic picture, composed of fever, cough, asthenia, adynamia, myalgia and dyspnea
(Rodriguez-Morales et al., 2020; Fu et al., 2020). However, other studies report that the initial presentation may debut with isolated pneumonia, sudden respiratory failure, myocardial injury, renal failure, encephalitis, or stroke (Zhao et al., 2020). Ischemic stroke is a condition of severe brain injury that leads to high rates of morbidity, mortality and disability, in addition to high economic costs in terms of diagnostic, therapeutic and rehabilitation intervention (Tan et al., 2020).

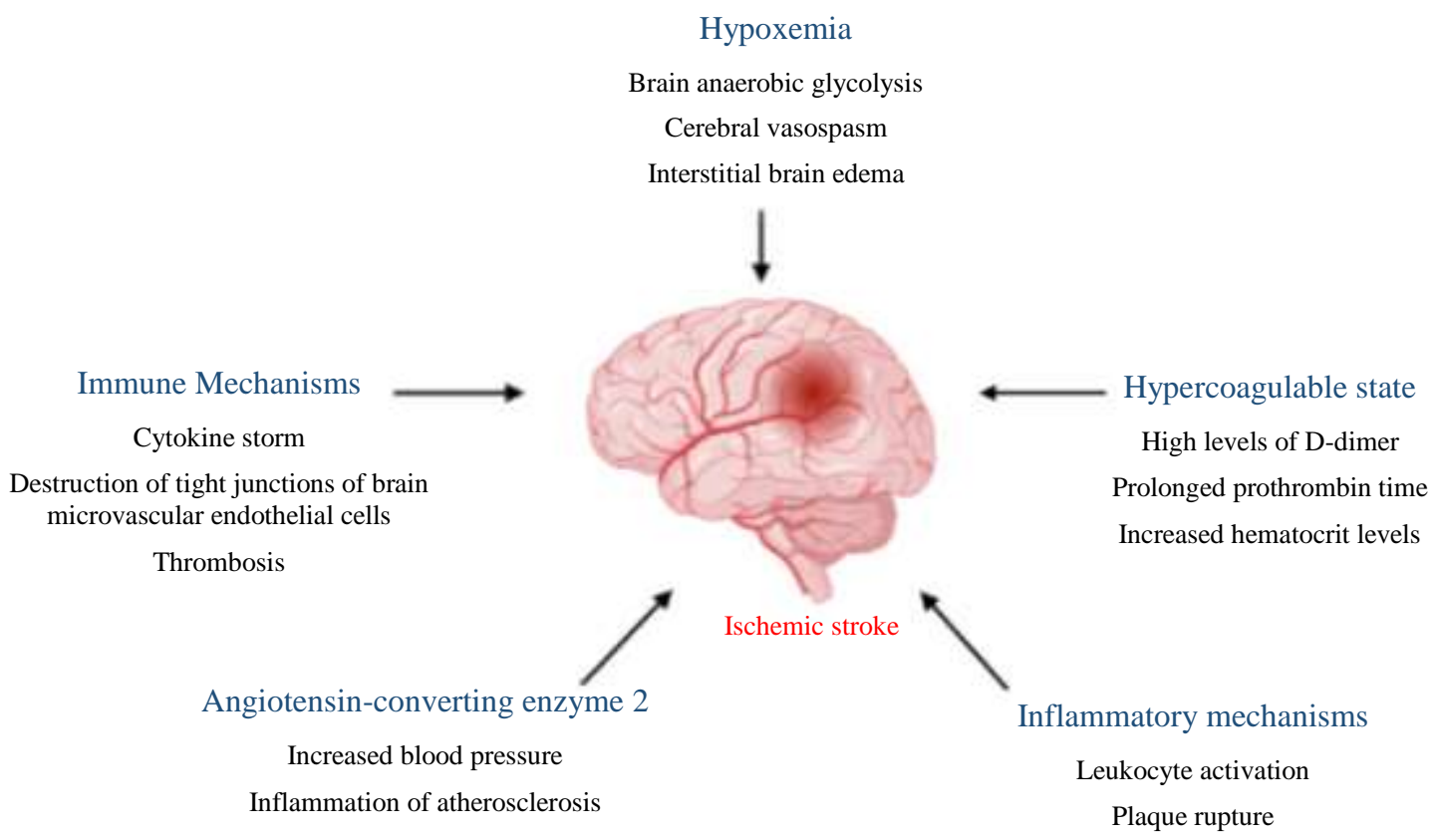

Fig. 1: Proposed pathophysiological mechanism on the development of ischemic stroke in COVID-19 patients. Inflammatory, immunological processes, hypercoagulability state, hypoxia and SARS-Cov 2/Angiotensin-Converting Enzyme 2 interaction are responsible for the central nervous system involvement. Therefore, all these mechanisms should be taken into account when establishing the prognosis and treatment of the patient with COVID-19. Created with BioRender 
It has been described that coronaviruses have tropism to the central nervous system, although the pathophysiological mechanism of their involvement is not entirely clear up to date (Morassi et al., 2020). The evidence of this tropism is controversial, because of the heterogeneity in the magnitude of deterioration found in the series of the evaluated cases (Rodriguez-Morales et al., 2020; Fu et al., 2020; Zhao et al., 2020), but clinical, laboratory, molecular and genetic findings have led to support that there is a clear tropism of coronaviruses to the central nervous system (Fan et al., 2020). Given the findings of lymphopenia, prolonged prothrombin time, high lactate dehydrogenase levels, neutrophilia, increased levels of D-dimer, creatinine, creatine-phosphokinase and oxygen therapy requirement, it is suggested that these patients undergo a progressive and persistent state of inflammation, hypoxia and hypercoagulability (Fig. 1), which would explain the sudden presentation of fatal complications such as ischemic stroke, acute respiratory distress syndrome, septic shock, among others (Qureshi et al., 2020; Fan et al., 2020). Specifically, at the level of the central nervous system, endothelial dysfunction (increased vascular vasoconstriction, lipid accumulation and apoptosis), leukocyte activation and thrombosis under inflammatory stimulation (Fan et al., 2020), compromise brain circulation and these are consistent with evidence that shows that patients with cardiovascular risk factors are more likely to develop ischemic stroke (Fu et al., 2020; Zhao et al., 2020; Tan et al., 2020; Morassi et al., 2020).

Tan et al. (2020) state that the incidence of ischemic stroke in COVID-19 patients ranges from 0.9 to $2.7 \%$, however, they maintain that the degree of involvement is moderate to severe, with a prevalence of about $41 \%$ of lesion of large vessels and a mortality of $38 \%$. On the other hand, (Morassi et al., 2020) also argue that stroke is a severe complication of COVID-19, which can occur even in patients who do not have cardiovascular risk factors. In their study, the evaluated patients had multisystem involvement (severe pneumonia, renal failure, liver failure, alterations in coagulation), $83 \%$ died and the remaining percentage was left with serial neurological sequelae.

The relevance of this topic lies in that its presentation can occur in both asymptomatic and symptomatic and in both cases, significantly increasing morbidity and mortality, therefore, the continuous evaluation of neurological functionality is an indispensable aspect in the approach of this disease, regardless of the risk possessed by the patient. This evidence should serve as a support for the strengthening of transport networks and specialized health care services, since there are no hemodynamics units aimed exclusively at COVID-19 patients in various places around the world, as well as fortified health systems, as the prognosis of those patients even with mild neurovascular involvement, can become nefarious at any time (Fan et al., 2020), due to delays or lack of equipment for the timely care of the ischemic event.

The consensus on the management of ischemic stroke in COVID-19 patients published by Qureshi et al, states that all asymptomatic patients with neurological symptoms (such as confusion, severe headache and dizziness), should be included in the stroke management protocol until proven otherwise (Qureshi et al., 2020). Emphasizing that the evaluation of these cases in the emergency department is not easy, due to the difficulties presented such as a poor medical history, as it is not possible to obtain clear data from the affected patient or the family member; the presence of neurological symptoms typical of the natural history of COVID-19 disease and the absence of signs and symptoms in cases with a mild injury. However, the clinical impact of knowing this condition as a possible complication, in any case, increases the possibility of making an early diagnosis through the use of screening instruments.

\section{Authors' Contributions}

All authors have contributed equally in this paper.

\section{Ethics}

In this article, ethical principles related to scientific research articles are observed. The corresponding author confirms that both authors have read, revised and approved the paper.

\section{References}

Fan, H., Tang, X., Song, Y., Liu, P., \& Chen, Y. (2020). Influence of COVID-19 on cerebrovascular disease and its possible mechanism. Neuropsychiatric Disease and Treatment, 16, 1359. https://doi.org/10.2147/NDT.S251173

Fu, L., Wang, B., Yuan, T., Chen, X., Ao, Y., Fitzpatrick, T., ... \& Zou, H. (2020). Clinical characteristics of coronavirus disease 2019 (COVID-19) in China: a systematic review and meta-analysis. Journal of Infection, 80(6), 656-665. https://doi.org/10.1016/j.jinf.2020.03.041

Morassi, M., Bagatto, D., Cobelli, M., D’Agostini, S., Gigli, G. L., Bnà, C., \& Vogrig, A. (2020). Stroke in patients with SARS-CoV-2 infection: case series. Journal of Neurology, 267, 2185-2192. https://doi.org/10.1007/s00415-020-09885-2

Qureshi, A. I., Abd-Allah, F., Al-Senani, F., Aytac, E., Borhani-Haghighi, A., Ciccone, A., ... \& Wang, Y. (2020). Management of acute ischemic stroke in patients with COVID-19 infection: Report of an international panel. International Journal of Stroke, $15(5)$, 540-554. https://doi.org/10.1177/1747493020923234 
Rodriguez-Morales, A. J., Cardona-Ospina, J. A., Gutiérrez-Ocampo, E., Villamizar-Peña, R., Holguin-Rivera, Y., Escalera-Antezana, J. P., ... \& Sah, R. (2020). Clinical, laboratory and imaging features of COVID-19: A systematic review and meta-analysis. Travel Medicine and Infectious Disease, $34, \quad 101623$. https://doi.org/10.1016/j.tmaid.2020.101623

Tan, Y. K., Goh, C., Leow, A. S., Tambyah, P. A., Ang, A., Yap, E. S., ... \& Tan, B. Y. (2020). COVID-19 and ischemic stroke: a systematic review and metasummary of the literature. Journal of Thrombosis and Thrombolysis, 50(3), 587-595. https://doi.org/10.1007/s11239-020-02228-y
Zhao, M., Wang, M., Zhang, J., Gu, J., Zhang, P., Xu, Y., ... \& Wan, J. (2020). Comparison of clinical characteristics and outcomes of patients with coronavirus disease 2019 at different ages. Aging (Albany NY), 12(11), 10070. https://doi.org/10.18632/aging.103298 